\title{
Design of a Mobile System for Metro Value-Added Services
}

\author{
Xian Zhu ${ }^{1,2, a}$, Linzhi Zhu ${ }^{1, b}$ \\ ${ }^{1}$ School of Health Economics \& Management, Nanjing University of Chinese Medicine, Nanjing, China \\ ${ }^{2}$ School of Information Management, Nanjing University, Nanjing, China \\ ae-mail: shellezhu@hotmail.com, be-mail: ailain_zu@foxmail.com
}

Keywords: Metro Value-added Services, Smart City, Mobile System, System Design

\begin{abstract}
In recent years, metro has not only become an important means of public transportation for local people, but also become a window to show a city's appearance and cultural features. In our research, we comprehensively considered the additional services metro passengers may require and designed a mobile system used for providing them value-added services of city metro. The application was designed after functional requirement conducted seriously as four basic function modules (bicycle rental, umbrella rental, book exchange and city information) was ultimately included. Since the city metro's attribute of cultural carrying and information sharing has not been fully exploited and applied, this study is valuable and practical for the public construction of a city and provided more convenience for citizens.
\end{abstract}

\section{Introduction}

In 2010, IBM formally put forward the vision of 'smart city'. A 'smart city' should make full use of information and communication technology methods as the key for information measurement, analysis, integration of city services, to create a better city life for citizens[1]. As a part of the public infrastructure of a city, metro is gradually popularized and widely used which plays a significant role in citizens' daily life. Apart from the basic transportation function it provide, more potential that metro can bring us is worth exploring deeply.

Thanks for the development of mobile communication technology, a variety of city services can be improved with the help of mobile phone applications nowadays. Based on mobile phone, users can conduct a series of operations such as information searching, mobile payment, social activities etc. As the metro is a kind of dynamic and travelling city service, the portable and location recognizable capability of mobile phone is adaptive to serve this transportation.

All over the world, the development of value-added services around the metro is diversified. In Paris of France, public bicycles are placed around the rail traffic and commercial centers to realize seamless connection of public traffic conversion. Vélib, the public bicycle service, launched a mobile app to open up a new channel for bicycle rental trough mobile terminals [2]. In 2011, Korean supermarket chain Home Plus launched virtual supermarket in metro to present virtual supermarket goods [3]. People obtain specific goods' information by scanning a QR code and accomplish payment operation through their mobile phones. In China, the number of metro value-added services is increasing, but very few combine with the mobile network, most of which are off-line mode. Some experts and institutions have found this issue and began to explore. For example, Beijing Metro's 'M library' provide passengers e-books for reading by scanning the QR code in the metro. In addition, bicycle rental services become more and more popular in many cities from the beginning of 2017. Alipay, the largest third party payment platform strive to create a 'sesame credit' for umbrella rental in Shanghai, Hangzhou and Tianjin.

However, the information services and interaction experience these services provide are not arrive the balance as they only focus on information presentation, but do not pay attention to information transmission and feedback. Besides, each of them only has a single function, but do not have a consciousness of combination of metro value-added services. The purpose of this study is to explore the possibility of intelligent portable city services and to design and develop a mobile 
system to provide metro value-added services which can serve as a part of the 'smart city' construction.

\section{Functional Requirement Analysis}

According to the purpose of this study, a questionnaire of value-added service systems for metro is designed. The questionnaire data were collected both online and off-line near the metro stations. From March 6, 2017 to April 7, 2017, for a period of 32 days, a total of 587 valid questionnaires were obtained.

As a result, the main issues of present metro value-added services are: 1) the imbalance between supply and demand; 2) tedious operation procedure; 3) lack of publicity or service facilities; 4) lack of intelligent positioning and feedback mechanism. In addition, the top five functions which capture users' interest are: umbrella rental, city characteristic information, bicycle rental, transfer route planning and book exchange.

This metro value-added system is a polymeric platform. According to users' interests, we choose bicycle rental, umbrella rental, book exchange, city characteristic information as the main functions of the system. Based on that, we give the system modules structure in Fig.1.

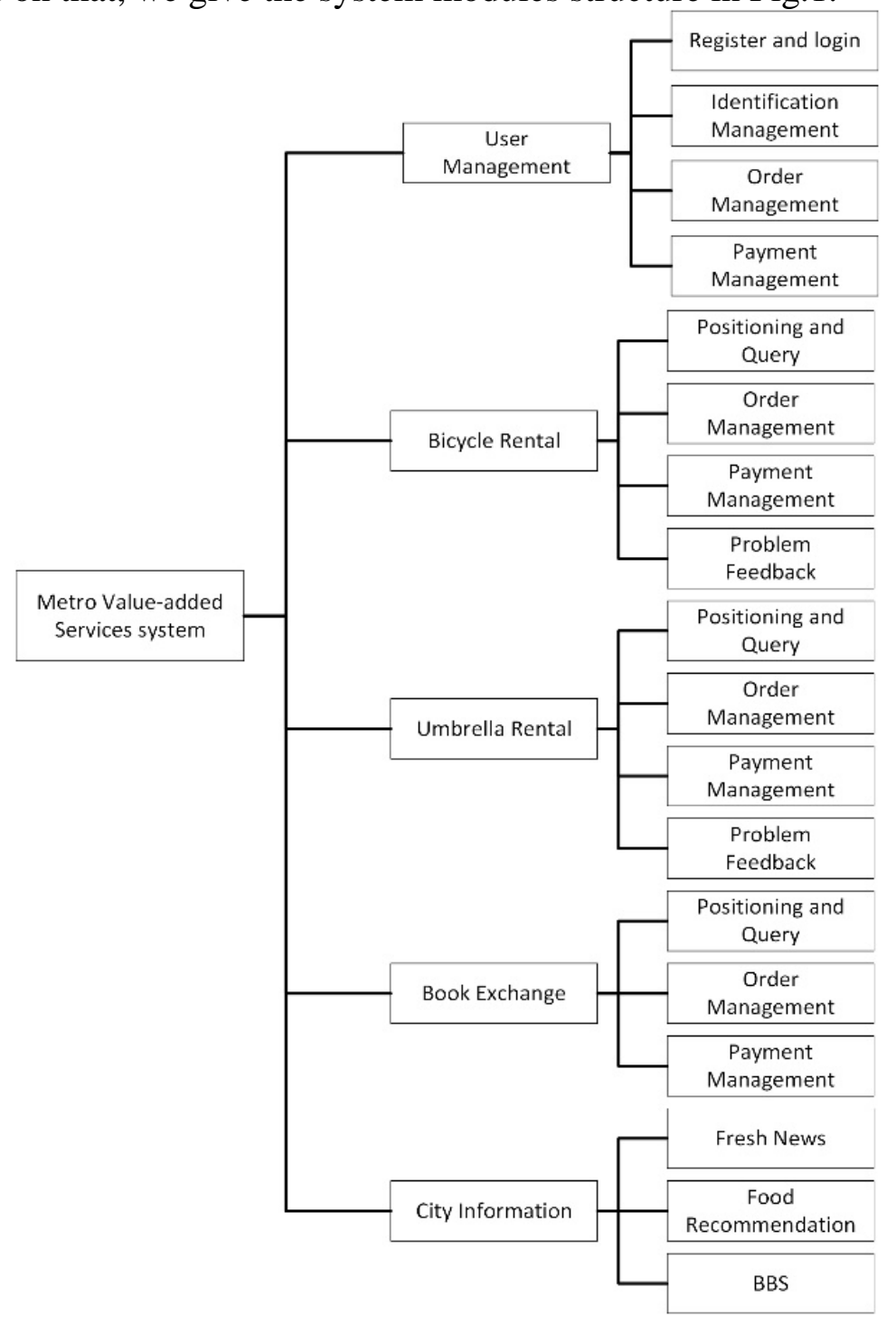

Figure 1. System functional modules

\subsection{User Management}

User management is the most basic part of the system. User management system includes user registration, user identity information management, order management and payment management. 
User login is the first step for using other functions of the system. Identity authentication is a necessary step for bicycle/umbrella rental and book exchange. Through identity information management, users can supplement or modify the authentication information. Order management part is where current user can query their order status. And the payment management part is for user to pay or withdraw deposit and check account information.

\subsection{Bicycle Rental}

The public bicycle information provided here is mainly the vehicles along the metro line. Bicycle rental function makes a good connection between the metro and public bicycles. This function includes four parts: bicycle query, order management, payment management and problem feedback. The user can manually enter the address or location, search the public bicycles near a specific metro station, and then make orders. Users can use the bicycle by enter the password the system provides and return the bicycle after usage. Deposit should be paid before rental and the rental fee should be paid after usage. The system supports the WeChat payment, Alipay payment methods. In order to regulate bicycle parking, the system is set up that parking to a specific location which the system identified can obtain an extra payment discount. In addition, user can report usage feedback anytime for better user experience.

\subsection{Umbrella Rental}

To bring more convenience to users travelling in rainy days, umbrellas in metro station can provide better city service. Users can inquire the number of remaining umbrella in every metro station and scan the code to borrow an umbrella. If the return period is nearly finished, the system will automatically send reminder to users. This function includes four parts: umbrella query, order management, payment management and problem feedback. According to the location selection or real-time positioning, the query can be made before users reach the certain station. Users scan the code to borrow an umbrella and return it to any station. If the deposit has been paid, umbrella can be taken and the deposit can be withdrew any time after user returns the umbrella. User feedbacks help us to repair timely and understand the users.

\subsection{Book Exchange}

Book exchange function enriches the social activities of the metro. Users can share books, magazines, newspapers etc. with other metro passengers. This function mainly includes three parts: book query, order management and payment management. User can upload their books' information into this system and search for another book other passenger share. Users can create, modify, delete, update their own book lists, search books, make orders and find a metro station to exchange books.

\subsection{City Information}

City information is an information combination of city news, food recommendation along the line, etc. These information can be optimized only based on the user's location to update information simultaneously. User can search city's food and travel information by enter the keywords or by select a certain station name. Meanwhile, they can publish information or communicate with other passengers.

\section{System Module Design and Interface Design}

When we were designing the prototype of this system, we determined and followed the interaction principles below:

1) Flat style: The whole system was designed in flat style with uniform color, removing the redundant, heavy and complicated decoration. Considering several functions of this system have the meaning of environmental protection, the color of blue green was selected.

2) Interfacial consistency: The consistency of the interface is not only reflected in the unity of the interface color, but also reflected in the consistency of the control position. In order to facilitate usage, the system placed commonly used function button on a fixed position, such as user 
information management will always be settled on the upper left corner.

3) Understandability of operation: The system followed the custom from top to bottom, from left to right when designing. Using familiar icons helps to understand the meaning of the buttons of search, return, home page, etc.

4) Controllability of operation: Users are the operators of the system. They are entitled to choose to continue or return according to their own situation. In the process of system design, we ensured that after each function item completed, they can click to the home page [4].

5) Explicit feedback: When there is an operation error, prompts will be showed. For example, when users miss filling in some options, red box striking tips will be presented on the top of the screen. When users' operations are completed, there will be corresponding prompt to inform the user to submit.

\subsection{Start and Home Page Design}

When users start this system, a three-second boot animation will be showed. This animation has the purpose to send a message to the user that the platform is starting which also helps to relieve waiting anxiety. Moreover, it is the first impression this system gives users which helps users understand the design concept and prepare for the contents they will meet later. (Fig. 2)

The design of the home page (Fig.3) is different from the traditional interface. Replacing the traditional box button, we chose more lively scenes instead. The selected characters and scenes and the entire software style are unified and harmonious. Besides, text description are put under the pictures to help users to understand.

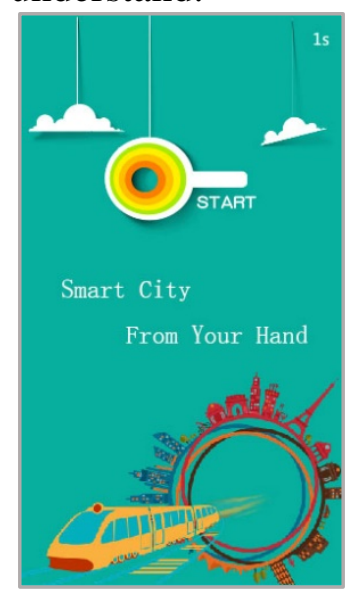

Figure 2. Start Page

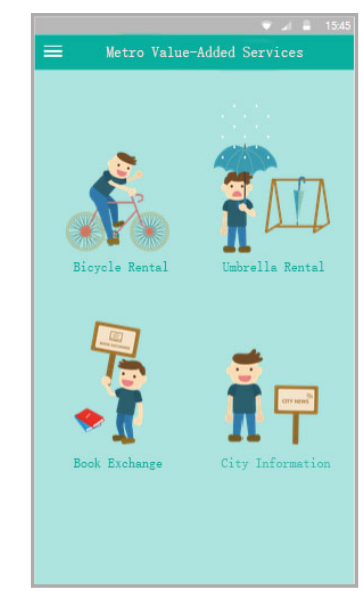

Figure 3. Home Page

\subsection{User Management Module Design}

This module has the property of strong flexibility and real-time capability which can be involved in the usage progress of various functions. Therefore, it was set as the resident module of the sidebar, which can be managed and checked by the users any time. When the user clicks on the button on the upper left corner, and the sidebar appears, including a list of 'personal center', 'order management', 'message reminder' and 'my wallet', which also can be closed when you don't need it. (Fig.4) 


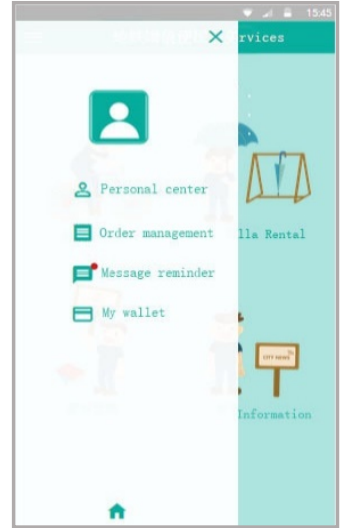

Figure 4. User management

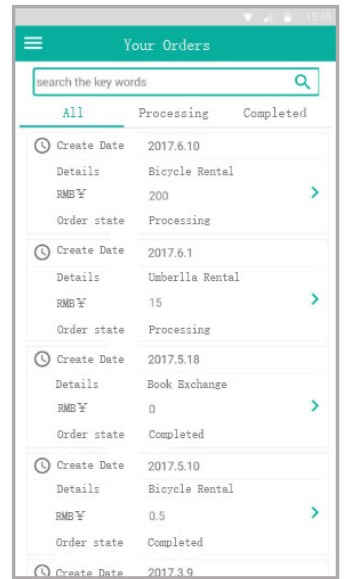

Figure 6. Order Management

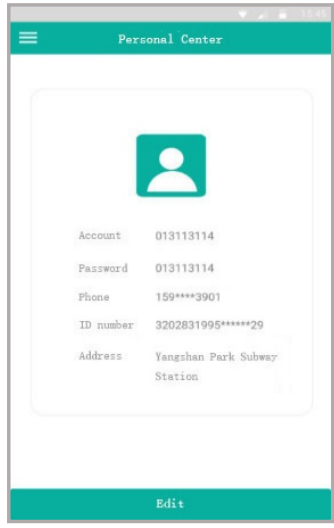

Figure 5. Personal Center

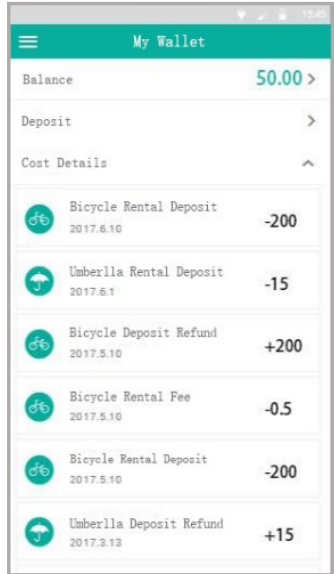

Figure 7. My wallet

\subsubsection{Personal Center}

By click on the 'personal center', there will show personal information which users can modify and save. (Fig.5)

\subsubsection{Order Management}

'order management' is a collection and combination of orders produced by the three main functions-bicycle rental, umbrella rental and book exchange. In 'order management', users can view the 'processing' or 'completed' orders through the status tab. In addition, users can search for orders directly through the search box. Click the order in the order list, details of the order will be presented. (Fig.6)

\subsubsection{Message Reminder}

Message reminder is an example of humanization design of the system. The system can conduct real-time detection of orders, provide timely feedback to users. The messages here may be an umbrella rental reminder, the feedback of book exchange, the reply of system or other users.

\subsubsection{My Wallet}

My wallet provides several payment methods. Users can pay deposit, view their balance and recharge in the wallet. In the process of rental, if users do not want to return the deposit immediately, they can save the money in the wallet. 'cost details' is where user can read the latest balance records. (Fig.7)

\subsection{Bicycle Rental Module Design}

Bicycle rental module includes bicycle query, bicycle taking, order management, payment management, repair feedback and other operations. 
Since the bicycle circulation is tremendous, bicycle searching use the map real-time positioning method. Although the design of the query is mainly aimed at metro bicycles, parking is not stationary. The data uploaded by map positioning can be more visualized for the bicycle management. (Fig. 8)

In addition, the large running cost of bicycle rental requires real-time billing. On the page we design here are two eye-catching lines-one is duration time and the other is the cost. (Fig.9)

Moreover, users need parking guidance and specification. One of the problems of sharing bicycles is the nonstandard and haphazard parking, which not only damages the environment of the city, but also hinder the ordinary traffic. Therefore, when the user wants to park, he/she clicks the 'stop' button, a prompt will be showed to guide the user park the bicycle in a reasonable area which can also bring himself/herself a rental discount.

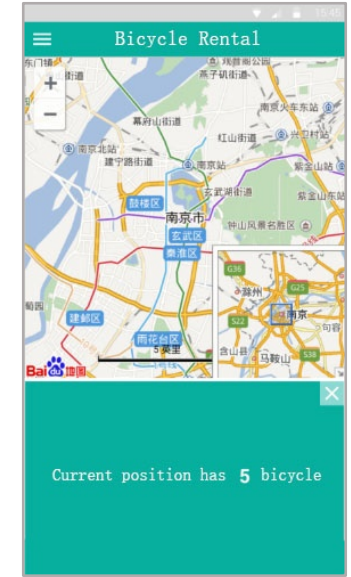

Figure 8. Bicycle query

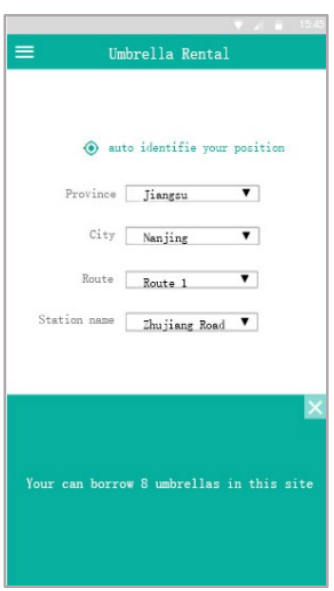

Figure 10. Umbrella query

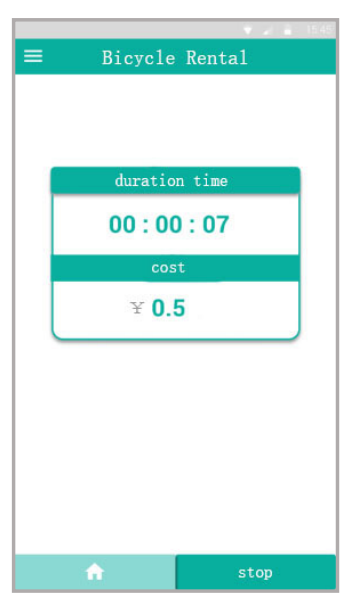

Figure 9. Time and billing

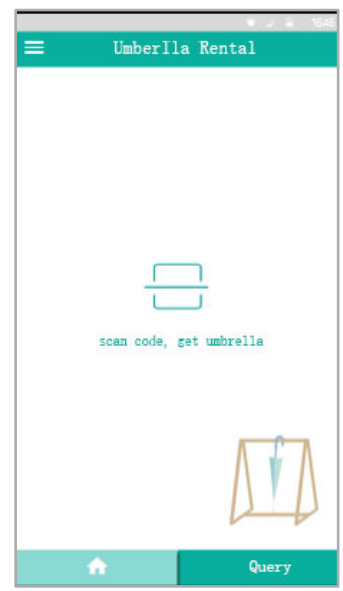

Figure 11. Code Scanning

\subsection{Umbrella Rental Module Design}

Umbrella rental module includes umbrella query, umbrella taking, order management, payment management and repair feedback etc. When a user selects the umbrella rental function, code scanning page will be displayed. If the user has arrived near the umbrella rental area, he/she can scan the code through a button click on the center of page, then take an umbrella. If the user has not arrived, he/she can conduct umbrella query by clicking the query button through system positioning automatically or by manual selection. (Fig.10, Fig.11)

After the code scanning, the system automatically identifies the position information and the specific number of the umbrella, meanwhile reads the user's personal information to generate an umbrella order. After the user's confirmation, the system will check if the deposit has been paid before, then the umbrella stand will automatically unlock an umbrella. The page prompts to show the rental is successful and the return deadline. If the user returns the umbrella within 7 days, the system changes the rental status to order completion. If it is due with the umbrella not been returned 
yet, the system will send reminders. In addition, on the order completion page, there is a feedback button for users to report problems occur when using umbrellas.

\subsection{Book Exchange Module Design}

Book exchange module mainly includes book query, book management, order management etc. The way users search books is very flexible as they can browse by the list of books or they can enter keywords by themselves (Fig. 12). Click on the book item to view book details. And the detail page shows the information of books and the book owners. When determining a book to read, users choose a book they own for exchange. They can select from the book library they created before or add a new book. The exchange request will be sent to the other book owner. After confirming the book exchange, the two users can exchange in a certain metro station or communicate through the contact information they provided before to this system.

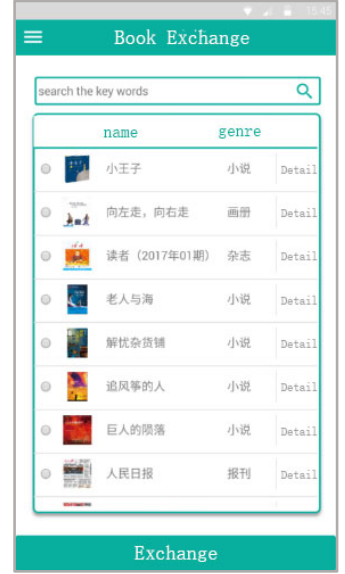

Figure 12. Book query

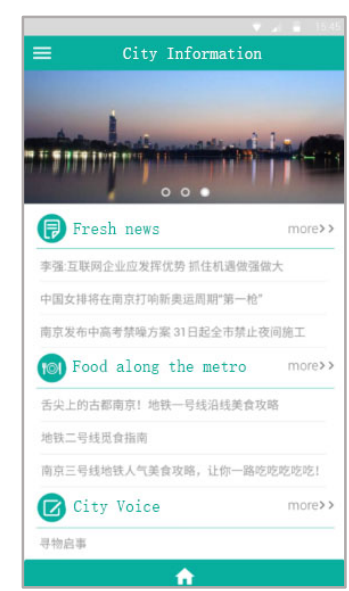

Figure 13. City information

\subsection{City Information Module Design}

This module is an information collection of the city. On the page carousel figures are set up to display the city features and recommendation. There are three sub-sections which are named 'Fresh news' and 'Food along the metro' and 'City voice' (Fig. 13). Considered to design the page viewable and enjoyable, each sub-section only display three pieces of information. Click more, more hidden contents will be found. On the content setting, 'Fresh news' and 'Food along the metro' are selected and published by the system manager while 'City voice' is where users post their information and communicate with each other. Click to 'see the details', they can participate to comment.

\section{Evaluation and Conclusion}

After completion of the system prototype design, we tested the function of each module of the system, compared with the requirements, and then improved the system design. The several aspects we refined are as below:

1) In the original design, the choice of the return path of the deposit was not clear. Now, the users have unambiguous and multiple choices.

2) In terms of payment function, the payment method is limited, other payment method should be considered like on-line bank, credit card etc.

3) Book modification operation was added in book management section.

4) In the city information module, we improved messaging, sharing and chatting functions, promote the interaction and communication between users.

Nowadays, citizens have a high degree of dependence on using mobile phones and tend to embrace new things with tolerance and try attitude, which is a good opportunity of construction of smart city in this internet era. Metro value-added services system is smart and can bring convenience to citizens. The self-supported and humanized services of this system provide metro 
passengers new experiences about city public transportation. Meanwhile, the concept of environmental protection, energy saving, circular sharing, rich and colorful urban information, are all conducive to promote the process of smart city and improve citizens' recognition and sense of pride of the city. Based on the analysis and design of the software and the results of test and evaluation, the following conclusions can be drawn:

1) This system fills the vacancy of metro value-added services system in the market. It provides a more efficient and flexible way of rental for the public bicycle and public umbrella services, promotes resources reuse and integration of the second-hand books, and create an open platform for mutual understanding between the citizens and the public with the city they live.

2) System interface and interaction give the most intuitive feeling to users in the process of using. Therefore, a good user experience is a significant factor we considered when we design this system. We believe it can enhance the user's satisfaction and loyalty, and therefore to make the system more vigorous for long.

\section{References}

[1] Scuotto V, Ferraris A, Bresciani S. Internet of Things: Applications and challenges in smart cities: a case study of IBM smart city projects[J]. Business Process Management Journal, 2016(2).

[2] Rahul Nair, Elise Miller-Hooks, Robert C. Hampshire, et al. Large-Scale Vehicle Sharing Systems: Analysis of Vélib'[J]. International Journal of Sustainable Transportation, 2013, 7(1):85-106.

[3] Lee H J. Development of the anymarket system for regional retail store[J]. International Journal of Multimedia \& Ubiquitous Engineering, 2013, 8(2):181-188.

[4] Robinson S, Marsden G, Jones M. There's Not an App for That: Mobile User Experience Design for Life[J]. Hci, 2014:9-10. 